\title{
The neurophysiology of auditory hallucinations - a historical and contemporary review
}

\author{
Remko van Lutterveld ${ }^{1,2}$, Iris E. C. Sommer ${ }^{1,2}$ and Judith M. Ford ${ }^{3}$ \\ Department of Psychiatry, University Medical Center, Utrecht, Netherlands \\ 2 Rudolph Magnus Institute of Neuroscience, Utrecht, Netherlands \\ ${ }^{3}$ Department of Psychiatry, Veterans Affairs Medical Center, University of California, San Francisco, CA, USA
}

Edited by:

Anthony A. Grace, University of

Pittsburgh, USA

\section{Reviewed by:}

John J. Foxe, Albert Einstein College of

Medicine, USA

Peter Uhlhaas, Max Planck Institute for

Brain Research, Germany

*Correspondence:

Remko van Lutterveld, Department of

Psychiatry, University Medical Center

Utrecht, B01.206, Heidelberglaan 100,

3584 CX Utrecht, Netherlands.

e-mail: r.vanlutterveld@umcutrecht.nI

\begin{abstract}
Electroencephalography and magnetoencephalography are two techniques that distinguish themselves from other neuroimaging methodologies through their ability to directly measure brain-related activity and their high temporal resolution. A large body of research has applied these techniques to study auditory hallucinations. Across a variety of approaches, the left superior temporal cortex is consistently reported to be involved in this symptom. Moreover, there is increasing evidence that a failure in corollary discharge, i.e., a neural signal originating in frontal speech areas that indicates to sensory areas that forthcoming thought is self-generated, may underlie the experience of auditory hallucinations.
\end{abstract}

Keywords: EEG, MEG, auditory hallucination, corollary discharge, superior temporal cortex, schizophrenia, psychosis

\section{INTRODUCTION}

Electroencephalography (EEG) and magnetoencephalography (MEG) are neuroimaging techniques that distinguish themselves from other methods by their excellent temporal resolution. Both are neurophysiological techniques that allow investigators to track brain activity on a millisecond time-scale. EEG measures the electrical signals produced by groups of neurons in the brain, and MEG measures the concurrent magnetic signals elicited by these electrical signals. For source-localization, however, MEG may be a more suitable technique than EEG, as the electrical signals related to neuronal activity are smeared out by the skull, hampering accurate EEG source-localization. The magnetic signals measured by MEG are not substantially affected by the skull and can therefore be located more reliably. EEG, on the other hand, has two clear advantages over MEG: its accessibility to a large number of investigators, as EEG equipment is available at most hospitals, and its relatively low cost.

Many studies have used neurophysiological methods to study auditory hallucinations. Already in 1955, Sem-Jacobsen et al reported on brain activity related to hallucinations. Over time, several approaches have evolved. These approaches can be divided into five main paradigms, which we describe below.

The most intuitive strategy is to use symptom capture, in which patients indicate the presence of hallucinations, for example by button press. Brain activity during hallucinations is then compared to hallucination-free periods. A second approach is to combine symptom capture with event-related potentials (ERPs ${ }^{1}$ ) to assess the processing of auditory information during the active "state" of an auditory hallucination. A third approach associates ERPs with the tendency or the "trait" to hallucinate. In this approach the severity of hallucinations is correlated with an index of auditory processing. In a fourth approach, repetitive transcranial magnetic stimulation (rTMS, a method that applies mag-

${ }^{1}$ Event-related potentials are measured with EEG. Its MEG counterparts are called event-related fields (ERFs). netic pulses to the brain in order to activate or deactivate brain activity) has been used to study EEG correlates of auditory hallucinations. A fifth approach is to study basic neurophysiological mechanisms that may underlie the tendency to hallucinate. Each of these paradigms will be described in detail together with a summary of the findings.

\section{SYMPTOM CAPTURE STUDIES \\ EARLY SYMPTOM CAPTURE STUDIES}

Before the era of antipsychotic medications, depth electrocorticography (ECoG) studies were sometimes conducted in conjunction with neurosurgery for relief of severe psychotic symptoms. In one such ECoG study, Sem-Jacobsen et al. (1955) reported: "A close relationship between the patient's acute episodes of psychotic behavior and the electric activity was found. The findings in this study draw attention to the presence of focal spike discharges in some chronically psychotic patients during episodes of disturbance or hallucinations or both and to the presence of changes in the activity of the temporal lobe and probably the frontal lobe during hallucinations."

Thirteen years later, Marjerrison et al. (1968) used scalp recorded EEG for the first time to capture the electrophysiological signal associated with auditory hallucinations. They reported that newly admitted or readmitted acute schizophrenia patients who experienced hallucinations during the experiment had lower variation in mean integrated amplitude of the EEG than similar patients who were not hallucinating during the experiment. In the next two decades, EEG studies investigating hallucinations were scarce. In the 1970s, Whitton et al. (1978) recorded the spectral power preceding an auditory hallucination in six unmedicated patients. This was compared to EEG power preceding a response in healthy controls performing tests of creativity. They reported that EEG power was predominant in the delta and theta bands in the 4-s interval prior to reports of hallucinations and creative responses, and suggested that the intrusiveness of the hallucinatory experience may be similar to the sudden internal experience of solving a creative task. 
In a telemetry study, Stevens et al. (1979) equipped schizophrenia patients with EEG electrodes, and the EEG signal was sent through radio-waves to a base-station. With this system, the patients were able to walk freely about the ward or dayroom. Hallucinatory behavior (e.g., muttering) was coded by a trained observer, enabling the comparison of hallucination episodes with non-hallucination episodes ("symptom-capture"). In this study, Stevens and her team published EEG recordings of a hallucinating patient, reporting power increases during hallucinations in all frequency bands and scalp derivations with the exception of alpha in the left temporal region. In a follow-up study using the same paradigm, Stevens and Livermore (1982) reported that hallucinations correlated with the presence of ramp spectra in the EEG, i.e., spectra characterized by a smooth decline in power from lowest to highest frequencies. According to the authors, such spectra have previously been found in conjunction with subcortical spike activity of epilepsy, suggesting hallucinations were present subsequent to some abnormal subcortical discharge. However, ramp spectra can also emerge from eye and body movement, and the authors acknowledge that this may have influenced results. In general, EEG data from patients in an uncontrolled environment should be interpreted with caution, because of muscle artifacts potentially confounding results.

A few years later, Serafetinides et al. (1986) investigated the influence of verbal versus button-press methods to indicate auditory hallucinations on oscillations in the EEG. The method used to determine the presence of hallucinations had a marked effect on EEG results. Verbal reporting was associated with a bilateral increase of high frequency activity, while non-verbal reporting was associated with an asymmetry in power between the left and right hemisphere. After this publication, no study made use of verbal reporting of hallucinations anymore.

\section{CONTEMPORARY SYMPTOM CAPTURE STUDIES}

With the advent of better analysis algorithms and greater computer power, EEG and MEG data can be decomposed into precise information in the time-frequency domain, while also providing better spatial resolution than the older clinical EEG methods. However, modern EEG and MEG symptom capture studies investigating hallucinations are scarce. To date, only one EEG study and three MEG studies have been published. In the EEG study, Sritharan et al. (2005) reported an increase in alpha band power in the left superior temporal cortex during auditory hallucinations in seven schizophrenia patients. Moreover, an increase in synchronization between the left and right superior temporal cortices was found during auditory hallucinations, suggesting an increase in functional coupling between these brain regions during hallucinations.

Ishii et al. (2000) were the first to investigate auditory hallucinations using MEG in a symptom capture design. In a case-study they reported an increase in theta-band activity in the left superior temporal cortex during hallucinations. In another case-study, the same structure was implicated, albeit in the beta band (Ropohl et al., 2004). Reulbach et al. (2007) studied five patients with nonverbal auditory hallucinations (e.g., noise, music) and three patients with command hallucinations. Hallucinations in the former group were associated with an increase in beta-band activity in the left superior temporal cortex, while hallucinations in the latter group were associated with the same activation pattern extending into the left dorsolateral prefrontal cortex. According to the authors, these findings suggest that the lack of frontal lobe involvement in nonverbal hallucinations could be interpreted as a sign of diminished cortical involvement compared to the complex mechanisms involved in the generation of voices. In sum, heterogeneous results regarding involved frequency bands were observed. The theta, alpha, and beta bands were all reported to be implicated in the experience of auditory hallucinations. A possible explanation for these heterogeneous results could be the small sample sizes of the studies (see Table 1), as studies with small sample sizes lead to more variable and less reliable results. Results regarding location were more consistent, as all studies showed increases in power in the left superior temporal gyrus (STG).

\section{COMBINED ERP/ERF-SYMPTOM CAPTURE STUDIES}

Another approach to study auditory hallucinations is to combine symptom capture with ERPs. ERPs are evoked by a stimulus, and the components of interest usually occur within one second after stimulus-presentation. With the combined symptom-capture-ERP method, ERPs are studied during hallucinatory periods and compared to ERPs during non-hallucinatory periods. A frequently used ERP in this approach is the N100 component. The N100 is generated in the auditory cortex (Hari et al., 1984), and is considered to be a standard metric of auditory cortex activation. As such, the N100 provides the opportunity to compare auditory cortex activity during the hallucinatory state with the non-hallucinatory state. Tiihonen et al. (1992) measured N100 amplitude and latency to tones presented to two patients suffering from intense auditory hallucinations. In both patients, the N100 was delayed during the experience of auditory hallucinations compared to when the patients were not hallucinating. Moreover, in one patient N100 amplitude was also lower during hallucinations. In a larger study, Hubl et al. (2007) investigated N100 amplitude in seven patients with a psychotic disorder with acute auditory hallucinations, and found smaller amplitudes during hallucinations. Moreover, the largest differences in N100 source strength between periods

Table 1 | Summary of contemporary symptom capture studies.

\begin{tabular}{|c|c|c|c|}
\hline Study & Technique & $N$ & Findings \\
\hline Sritharan et al. (2005) & EEG & 7 & $\begin{array}{l}\text { Increase in power in the left } \\
\text { STG (alpha-band) } \\
\text { Increase in bitemporal } \\
\text { coherence (alpha-band) }\end{array}$ \\
\hline Ishii et al. (2000) & MEG & 1 & $\begin{array}{l}\text { Increase in power in the } \\
\text { left STG (theta-band) }\end{array}$ \\
\hline Ropohl et al. (2004) & MEG & 1 & $\begin{array}{l}\text { Increase in power in the } \\
\text { left STG (beta band) }\end{array}$ \\
\hline \multirow[t]{2}{*}{ Reulbach et al. (2007) } & MEG & 5 & $\begin{array}{l}\text { Increase in power in the left } \\
\text { STG during auditory non-verbal } \\
\text { hallucinations (beta band) }\end{array}$ \\
\hline & & 3 & $\begin{array}{l}\text { Increase in power in the left } \\
\text { STG extending into the left } \\
\text { DLPFC during auditory verbal } \\
\text { hallucinations (beta band) }\end{array}$ \\
\hline
\end{tabular}

STG, superior temporal gyrus; DLPFC, dorsolateral prefrontal cortex. 
with and without hallucinations were located in the left superior temporal cortex. The authors concluded that these findings indicate competition between auditory stimuli and auditory hallucinations for physiological resources in the primary auditory cortex, and that abnormal activation of this brain region could be a component of auditory hallucinations.

Line et al. (1998) took advantage of the rapid time-scale of EEG to study the time-frame surrounding auditory hallucinations. They presented eight schizophrenia patients with flickering visual stimuli, leading to the generation of electrical activity in the brain at the same frequency of the flashing stimulus (so-called steady-state visual evoked potentials). In the second before the onset of an auditory hallucination, patients showed a large and significant decrease in latency of brain responses in the right temporoparietal area, suggesting involvement of this area in the genesis of hallucinations.

In a very recent EEG study, transiently stable neuronal states were investigated (Kindler et al., 2010). Kindler et al. found that a so-called microstate associated with error monitoring was shorter during hallucinatory periods compared to non-hallucinatory periods. The authors speculated that the early termination of this microstate facilitated the misattribution of self-generated inner speech to external sources during hallucinations.

Like the contemporary symptom capture studies, combined ERP/ERF-symptom capture studies have small sample sizes (Table 2). Symptom capture studies are challenging since patients are required to hallucinate intermittently and for a considerable time of the experiment, and patients have to be able to reliably indicate onset and offset of their hallucinations (Ford et al., 2009).

\section{ASSOCIATIONS BETWEEN HALLUCINATORY TRAIT AND EEG/ MEG MEASURES}

Another strategy to study hallucinations is to investigate the association between EEG and MEG measures and the tendency to hallucinate. Lee et al. (2006) used quantitative EEG and source imaging to investigate 25 schizophrenia patients with treatment-refractory auditory hallucinations and 23 schizophrenia patients who were

Table 2 | Summary of combined ERP/ERF-symptom capture studies.

\begin{tabular}{|c|c|c|c|}
\hline Study & Technique & $N$ & Findings \\
\hline $\begin{array}{l}\text { Tiihonen } \\
\text { et al. (1992) }\end{array}$ & EEG and $\mathrm{MEG}$ & 2 & $\begin{array}{l}\text { Delay in N100 amplitude } \\
\text { during hallucinations } \\
\text { (both patients) } \\
\text { Smaller N100 amplitude } \\
\text { during hallucinations } \\
\text { (one patient) }\end{array}$ \\
\hline $\begin{array}{l}\text { Hubl } \\
\text { et al. (2007) }\end{array}$ & EEG & 7 & $\begin{array}{l}\text { Smaller N100 amplitude } \\
\text { during hallucinations }\end{array}$ \\
\hline $\begin{array}{l}\text { Line } \\
\text { et al. (1998) }\end{array}$ & EEG & 8 & $\begin{array}{l}\text { Decrease in latency in brain } \\
\text { responses to flickering } \\
\text { stimuli in the right temporoparietal } \\
\text { area in the second before } \\
\text { hallucination onset }\end{array}$ \\
\hline $\begin{array}{l}\text { Kindler } \\
\text { et al. (2010) }\end{array}$ & EEG & 9 & $\begin{array}{l}\text { Shorter microstate in the EEG } \\
\text { related to error monitoring } \\
\text { during hallucinations }\end{array}$ \\
\hline
\end{tabular}

hallucination-free for at least 2 years. Resting-state EEG in the hallucinating patients showed significantly increased beta-band activity in the left inferior parietal lobule and the left medial frontal gyrus compared to non-hallucinating patients. Moreover, gamma and beta frequencies were significantly correlated in hallucinating patients, but not in non-hallucinating patients. The authors suggested that the strong correlation between gamma and beta frequency oscillations may indicate that the brains of hallucinating patients act as if they were experiencing "real" auditory stimulation, as previous studies have shown strong correlations between gamma and beta frequency oscillations in normal populations in response to auditory stimuli (e.g., Haenschel et al., 2000).

Several authors have used ERPs to study associations with auditory hallucinations. Still, the relationship between ERPs and clinical symptoms of schizophrenia remains controversial. Havermans et al. (1999) studied the P3b evoked potential, which is regarded as a standard measure of effortful attention. The authors reported reductions in $\mathrm{P} 3 \mathrm{~b}$ amplitude in chronic hallucinating patients compared to non-hallucinating patients. Turetsky et al. (1998) found a strong association between a frontal P3b subcomponent and severity of auditory hallucinations. However, other studies failed to find any association between $\mathrm{P} 3 \mathrm{~b}$ amplitude and positive symptoms (Eikmeier et al., 1992; Liu et al., 2004). As most schizophrenia patients with auditory hallucinations also experience other symptomatology like delusions, some degree of disorganization, and negative symptoms, the diverse $\mathrm{P} 3 \mathrm{~b}$ findings may be related to this diversity in symptoms. In addition, antipsychotic medication may have affected the results.

To circumvent these problems, van Lutterveld et al. (2010) investigated P3b amplitude in non-schizophrenic individuals with auditory verbal hallucinations as an isolated symptom. These individuals functioned at normal professional and social levels and were free of medication. If the P3b amplitude reduction typically seen in schizophrenia patients (Jeon and Polich, 2003) is due to the tendency to hallucinate, then non-schizophrenic subjects who hallucinate should also have reduced $\mathrm{P} 3 \mathrm{~b}$ amplitudes. Contrary to expectations, they found an increase in $\mathrm{P} 3 \mathrm{~b}$ amplitude, which was interpreted as refuting a pivotal role of decreased effortful attention in the pathophysiology of auditory verbal hallucinations.

Finally, one study investigated the P3a ERP to speech sounds in hallucinating and non-hallucinating patients with schizophrenia. Unlike the P3b, the P3a is not associated with effortful attention, but with involuntary shifts to auditory changes and processing of novelty. Fisher et al. (2010) found that hallucinating patients had smaller P3a amplitudes than non-hallucinating patients, and that for the hallucinating patients $\mathrm{P} 3 \mathrm{a}$ amplitude was negatively correlated with auditory-hallucination symptomatology. The authors suggested that auditory verbal hallucinations are associated with impaired processing of external speech sounds, perhaps due to competition between external and internal auditory verbal stimuli (i.e., hallucinations).

Other studies have investigated mismatch negativity (MMN) and hallucinations. MMN is an ERP related to automatic auditory change detection. However, like $\mathrm{P} 3 \mathrm{~b}$ findings, results are inconsistent. Some studies reported an association between MMN amplitude and auditory hallucinations (Youn et al., 2003; Fisher et al., 2008a,b), while others did not (Schall et al., 1999; Kasai et al., 2002). 
These diverse findings may be at least partly explained by the different methodologies used. For instance, Schall et al. presented visual and auditory stimuli simultaneously, while others did not.

Recently, interest has been growing in auditory steady-state evoked potentials elicited by click trains. With this paradigm, a steady stream of clicks is presented (hence steady-state), and the brain's responses are measured over the presentation epoch (Uhlhaas and Singer, 2010). Spencer et al. (2009) presented click trains pulsing at $40 \mathrm{~Hz}$ to patients and healthy controls. They found that patients with higher gamma-band activity $(\sim 40 \mathrm{~Hz})$ in the left primary auditory cortex had a greater liability for experiencing auditory hallucinations. Moreover, this activity was influenced by delta-wave activity. The authors raise the possibility that aberrant oscillatory synchronization in the temporal cortex could interact with dysfunctional corollary discharge mechanisms (i.e., a malfunctioning in neural signals originating in frontal speech areas that indicate to sensory areas that forthcoming thought is selfgenerated), leading to the experience of auditory hallucinations. The reported correlations in this study were based on lifetime hallucination ratings, and the medicated patients were not actively hallucinating at the time of the study. Still, these findings extended earlier results of this laboratory, in which a correlation between gamma-band activity and hallucination severity of first-episode psychosis patients was found (Spencer et al., 2008).

\section{NEUROPHYSIOLOGY AND REPETITIVE TRANSCRANIAL MAGNETIC STIMULATION}

In the last decade, rTMS has emerged as a new potential treatment option for auditory hallucinations. With rTMS, electromagnetic induction is used to non-invasively increase or decrease brain activity. Two studies have investigated the effect of rTMS on the EEG in the context of auditory hallucinations. Jandl et al. (2006) reported that a subgroup of patients benefited from rTMS over the left superior temporal cortex as revealed by a decrease in auditoryhallucination severity, while no changes in whole-head EEG were reported. Horacek et al. (2007) applied rTMS to the left temporoparietal cortex for 10 days and reported a significant improvement in hallucination severity. TMS treatment caused a decrease in activity in the beta- 1 and -3 bands in the left temporal lobe, whereas an increase was found for the beta- 2 band in the right temporal cortex and the inferior parietal lobule, indicating transcallosal signal transmission involvement. A possible explanation for the divergent findings of the two studies is that data-analysis procedures were different. In the former study the EEG was assessed on sensor-level and in the latter study a source-localization procedure was used.

\section{STUDIES OF A BASIC NEURAL MECHANISM THAT MIGHT UNDERLIE AUDITORY HALLUCINATIONS}

Feinberg (1978) suggested that malfunctioning of the corollary discharge mechanism might underlie the experience of auditory hallucinations. Corollary discharge is a basic feed-forward system involved in suppressing the sensory consequences of self-generated actions (Sperry, 1950; Von Holst and Mittelstaedt, 1950). It has been documented across the animal kingdom (Crapse and Sommer, 2008), and its action allows all species to suppress sensations that result from their own actions and to tag them as coming from the self. Such feed-forward systems have been well described in the visual and somatosensory systems, but also serve the auditory system across species from crickets (Poulet and Hedwig, 2002) to song-birds (McCasland and Konishi, 1981) to non-human (Eliades and Wang, 2003), and human primates (e.g., Paus et al., 1996; Ford et al., 2007b). Because the corollary discharge mechanism operates on a rapid time-scale, this theory has most extensively been investigated using neurophysiological recordings. In humans, EEG (Ford et al., 2010) and MEG (Curio et al., 2000; Houde et al., 2002) have been used for studies of the auditory system; only EEG-based methods have been used in studies of schizophrenia.

While this mechanism explains suppression and tagging of sensations resulting from overt motor acts, Feinberg (1978) suggested that thinking may conserve and utilize the computational and integrative mechanisms that evolved for physical movement. In a well-functioning corollary discharge system a signal is sent from frontal areas involved in inner speech generation to temporal speech reception areas, tagging the perception as self-generated. When this mechanism is malfunctioning, a person may experience an auditory hallucination through misperceiving his or her own thoughts as being externally generated.

Several lines of research support the hypothesis of corollary discharge dysfunction in schizophrenia. The first line explored whether this system is deviant in schizophrenia patients versus healthy controls. In these studies, control subjects and patients first uttered syllables and then listened passively to a recording of that speech played back. EEG was recorded during both talking and listening conditions, and the amplitude of the N100 component of the ERP to speech onset was used as a measure of auditory cortical responsiveness. Consistent with the action of the corollary discharge system, N100 amplitude was smaller during talking than listening in healthy controls. Interestingly, there was significantly less N100 suppression in patients, suggesting aberrations in the corollary discharge system (Ford et al., 2001a, 2007a,b). In another N100 study, the effects of thinking on auditory cortical responsiveness were investigated. It was shown that thinking affected N100 amplitude in healthy controls, but not in schizophrenia patients (Ford et al., 2001b).

In a second line of research, functional connectivity, as measured by EEG coherence between frontal and temporal lobes in the gamma band, was found to be higher during talking than during listening in healthy controls. This pattern was disrupted when the uttered syllables were pitch-shifted while the subjects were talking, resulting in a non-self experience of the spoken sounds. In schizophrenia patients, distortion of the auditory feedback did not result in alteration of gamma-band frontotemporal coherence, again suggesting a malfunctioning corollary discharge system (Ford and Mathalon, 2005). In another coherence study, it was found that theta-band frontotemporal coherence was higher for talking than for listening for controls, but not for schizophrenia patients. This effect was carried by the hallucinating patients, as the nonhallucinators tended to show the pattern seen in the healthy controls. The authors suggested that a failure in the frontal-temporal network during overt speech may also occur during covert speech, leading to misattribution of self-generated thoughts to external sources (Ford et al., 2002). Given that N100 recorded from auditory 
cortex is suppressed during talking, the net result of coherent communication between frontal and temporal lobes was to suppress auditory sensation.

The corollary discharge theory can also be investigated by examining the small time-frame before speech starts. In such a study, pre-speech neural synchrony was reported to be related to subsequent suppression of N100 amplitude in healthy controls, but not in patients. Moreover, time-frequency analyses showed greater prespeech synchrony in healthy controls than in patients, especially in those with severe auditory hallucinations. The authors interpreted these findings as suggesting that EEG synchrony preceding speech reflects the action of the corollary discharge system, which dampens auditory responsiveness to self-generated speech and is deficient in patients who hallucinate (Ford et al., 2007b).

Another line of research explored the influence of pitch-shifting auditory stimuli on auditory cortex activation. In this paradigm, hallucinating schizophrenia patients, non-hallucinating schizophrenia patients, and controls were asked to utter meaningless sounds. Simultaneously, they were presented with auditory feedback of the uttered sounds, or pitch-shifted feedback of the uttered sounds, or feedback of sounds uttered by someone else, or pitchshifted feedback uttered by someone else. It was found that N100 amplitude to the unaltered self-voice was dampened relative to the altered self-voice or the alien auditory feedback. This pattern was not seen in hallucinating patients, and this imprecision correlated with the severity of hallucinations (Heinks-Maldonado et al., 2007).

Finally, in a recent study, subjects were asked to initiate auditory stimuli by button-press. It was found that N100 suppression was normalized in patients after adding a delay of $50 \mathrm{~ms}$ in the presentation of the stimulus, suggesting a temporal delay in corollary discharge (Whitford et al., 2011). Moreover, this normalization correlated with white-matter integrity of the arcuate fasciculus, a fiber bundle connecting speech/motor initiation areas in the frontal lobe with the auditory cortex in the temporoparietal lobe. These data suggest that structural deficits of the arcuate fasciculus may lead to temporally delayed corollary discharges, and that abnormalities in this fiber tract may be involved in the pathophysiology of auditory hallucinations.

\section{NEUROPHYSIOLOGY AND HALLUCINATIONS - WHAT DOES IT TELL US?}

Neurophysiological methods have been used in many different ways to understand auditory verbal hallucinations. The symptom capture approach has intuitive appeal and face validity, but it is operationally difficult and does not provide insight into the mechanisms by which voices might be heard. However, symptom capture studies do provide a wealth of information regarding the neural activity associated with the experience of hallucinations. Unfortunately, there is little consistency regarding the frequency of neural activity invoked by the hallucinatory experience, with evidence for increases in theta, alpha, and beta band activity associated with hallucinations. There is more consistency regarding the location of the activity invoked, with evidence that structures in the left temporal lobe are more active during a hallucination period than a non-hallucination period. Similarly, although ERPs elicited by probes during a hallucination provide little information about mechanisms, they do provide excellent support for the involvement of auditory cortex in the generation of the hallucination. This is in line with structural and functional magnetic resonance imaging (sMRI and fMRI) studies, in which left superior temporal cortex is implicated in the experience of auditory hallucinations (Barta et al., 1990; Dierks et al., 1999; Allen et al., 2008; Diederen et al., 2010).

Event-related potential studies of the trait to hallucinate suggest that the tendency to hallucinate is associated with reduced resources available for processing the probe. This is consistent with fMRI studies comparing hemodynamic activation to external probes in hallucinators and non-hallucinators (Ford et al., 2009), in which left temporal activation to auditory probes was reduced in patients who hallucinate compared to patients who do not. One EEG study reported greater beta band activity in the left inferior parietal lobe in patients who tended to hallucinate. Given the poor spatial resolution of EEG, it is possible that the increase in beta power was due to activity in the left auditory cortex. Finally, rTMS has provided indirect evidence of the involvement of left auditory cortex in hallucinations: In one study, treatment with rTMS over the left superior temporal cortex caused a decrease in activity in the beta band in the left temporal lobe with clinical improvement in hallucinations.

\section{FUTURE DIRECTIONS}

While mechanistic studies lack the intuitive face validity of the symptom capture work, they converge on the involvement of the temporal cortex in the generation and experience of auditory hallucinations, consistent with the report that voices sound loud and real. Mechanistic studies also offer translation to bench neuroscience and translation to other species, and hence open the door to invasive manipulations that are not possible with in vivo human studies. For one example, studies like the ones reviewed above can be applied in the future to animals who make social calls, such as song-birds (Brainard and Doupe, 2000) and non-human primates (Eliades and Wang, 2003). In such an experiment, perturbations of the neurotransmitters implicated in schizophrenia might produce a pattern in the neural signature of the mechanism that resembles the pattern seen in schizophrenia patients who hallucinate. Because symptom capture studies are infeasible in animal models, and because the relationship between the experience of auditory hallucinations and related frequency bands is unclear, we suggest that future research exploit a mechanistic approach in animal models of schizophrenia.

\section{ACKNOWLEDGMENTS}

Funding for this study was provided by the Dutch Science Organization (Nederlandse Wetenschappelijke Organisatie, NWO), Operating Grant: 916.56.172). NWO did not have any further role in the study design; in the collection, analysis, and interpretation of data; in the writing of the report; and in the decision to submit the paper for publication. Additional funding was provided by the Veterans Administration (Merit Review) and NIMH (R01MH076989 and K02MH067967).

\section{AUTHOR'S CONTRIBUTION}

Remko van Lutterveld wrote the first draft of the manuscript. I.E.C. Sommer supervised the study and Judith M. Ford co-wrote the manuscript and supervised the study. All authors contributed to and have approved the final manuscript. 


\section{REFERENCES}

Allen, P., Laroi, F., McGuire, P. K., and Aleman, A. (2008). The hallucinating brain: a review of structural and functional neuroimaging studies of hallucinations. Neurosci. Biobehav. Rev. 32, 175-191.

Barta, P. E., Pearlson, G. D., Powers, R. E., Richards, S. S., and Tune, L. E. (1990). Auditory hallucinations and smaller superior temporal gyral volume in schizophrenia. Am. J. Psychiatry 147, 1457-1462.

Brainard, M. S., and Doupe, A. J. (2000). Auditory feedback in learning and maintenance of vocal behaviour. Nat. Rev. Neurosci. 1, 31-40.

Crapse, T. B., and Sommer, M. A. (2008). Corollary discharge across the animal kingdom. Nat. Rev. Neurosci. 9 , 587-600.

Curio, G., Neuloh, G., Numminen, J., Jousmaki, V., and Hari, R. (2000). Speaking modifies voice-evoked activity in the human auditory cortex. Hum. Brain Mapp. 9, 183-191.

Diederen, K. M., Neggers, S. F., Daalman, K., Blom, J. D., Goekoop, R., Kahn, R. S., and Sommer, I. E. (2010). Deactivation of the parahippocampal gyrus preceding auditory hallucinations in schizophrenia. Am. J. Psychiatry 167, 427-435.

Dierks, T., Linden, D. E., Jandl, M., Formisano,E., Goebel, R.,Lanfermann, H., and Singer, W. (1999). Activation of Heschl's gyrus during auditory hallucinations. Neuron 22, 615-621.

Eikmeier, G., Lodemann, E., Zerbin, D., and Gastpar, M. (1992). P300, clinical symptoms, and neuropsychological parameters in acute and remitted schizophrenia: a preliminary report. Biol. Psychiatry 31, 1065-1069.

Eliades, S. J., and Wang, X. (2003). Sensory-motor interaction in the primate auditory cortex during selfinitiated vocalizations. J. Neurophysiol. 89, 2194-2207.

Feinberg, I. (1978). Efference copy and corollary discharge: implications for thinking and its disorders. Schizophr. Bull. 4, 636-640.

Fisher, D. J., Labelle, A., and Knott, V. J. (2008a). Auditory hallucinations and the mismatch negativity: processing speech and non-speech sounds in schizophrenia. Int. J. Psychophysiol. 70, 3-15.

Fisher, D. J., Labelle, A., and Knott, V. J. (2008b). The right profile: mismatch negativity in schizophrenia with and without auditory hallucinations as measured by a multi-feature paradigm. Clin. Neurophysiol. 119, 909-921.

Fisher, D. J., Labelle, A., and Knott, V. J. (2010). Auditory hallucinations and the P3a: attention-switching to speech in schizophrenia. Biol. Psychol. 85, 417-423.

Ford, J. M., Gray, M., Faustman, W. O. Roach, B. J., and Mathalon, D. H. (2007a). Dissecting corollary discharge dysfunction in schizophrenia Psychophysiology 44, 522-529.

Ford, J. M., Roach, B. J., Faustman, W. O., and Mathalon, D. H. (2007b). Synch before you speak: auditory hallucinations in schizophrenia. Am. J. Psychiatry 164, 458-466.

Ford, J. M., and Mathalon, D. H. (2005). Corollary discharge dysfunction in schizophrenia: can it explain auditory hallucinations? Int. J. Psychophysiol. $58,179-189$.

Ford, J. M., Mathalon, D. H., Heinks, T., Kalba, S., Faustman, W. O., and Roth, W.T. (2001a). Neurophysiological evidence of corollary discharge dysfunction in schizophrenia. Am. J. Psychiatry 158, 2069-2071.

Ford, J. M., Mathalon, D. H., Kalba, S., Whitfield, S., Faustman, W. O., and Roth, W. T. (2001b). Cortical responsiveness during inner speech in schizophrenia: an event-related potential study. Am. J. Psychiatry 158, 1914-1916.

Ford, J. M., Mathalon, D. H., Whitfield, S., Faustman, W. O., and Roth, W. T. (2002). Reduced communication between frontal and temporal lobes during talking in schizophrenia. Biol. Psychiatry 51, 485-492.

Ford, J. M., Roach, B. J., Jorgensen, K. W. Turner, J. A., Brown, G. G., Notestine, R., Bischoff-Grethe, A., Greve, D., Wible, C., Lauriello, J., Belger, A., Mueller, B. A., Calhoun, V., Preda, A., Keator, D., O’Leary, D. S., Lim, K. O., Glover, G., Potkin, S. G., and Mathalon, D. H. (2009). Tuning in to the voices: a multisite FMRI study of auditory hallucinations. Schizophr. Bull. 35, 58-66.

Ford, J. M., Roach, B. J., and Mathalon, D. H. (2010). Assessing corollary discharge in humans using noninvasive neurophysiological methods. Nat. Protoc. 5, 1160-1168.

Haenschel, C., Baldeweg, T., Croft, R. J., Whittington, M., and Gruzelier, J. (2000). Gamma and beta frequency oscillations in response to novel auditory stimuli: a comparison of human electroencephalogram (EEG) data with in vitro models. Proc. Natl. Acad. Sci. U.S.A. 97, 7645-7650.

Hari, R., Hamalainen, M., Ilmoniemi, R., Kaukoranta, E., Reinikainen, K., Salminen, J., Alho, K., Naatanen, R. and Sams, M. (1984). Responses of the primary auditory cortex to pitch changes in a sequence of tone pips: neuromagnetic recordings in man. Neurosci. Lett. 50, 127-132.
Havermans, R., Honig, A., Vuurman, E. F. Krabbendam, L., Wilmink, J., Lamers, T., Verheecke, C. J., Jolles, J., Romme, M. A., and van Praag, H. M. (1999). A controlled study of temporal lobe structure volumes and $\mathrm{P} 300$ responses in schizophrenic patients with persistent auditory hallucinations. Schizophr. Res. 38, 151-158.

Heinks-Maldonado, T. H., Mathalon, D H., Houde, J. F., Gray, M., Faustman, W. O., and Ford, J. M. (2007) Relationship of imprecise corollary discharge in schizophrenia to auditory hallucinations. Arch. Gen. Psychiatry 64, 286-296.

Horacek, J., Brunovsky, M., Novak, T., Skrdlantova, L., Klirova, M. Bubenikova-Valesova, V., Krajca, V. Tislerova, B., Kopecek, M., Spaniel, F., Mohr, P., and Hoschl, C. (2007). Effect of low-frequency rTMS on electromagnetic tomography (LORETA) and regional brain metabolism (PET) in schizophrenia patients with auditory hallucinations. Neuropsychobiology 55 132-142.

Houde, J. F., Nagarajan, S. S., Sekihara, K., and Merzenich, M. M. (2002). Modulation of the auditory cortex during speech: an MEG study. J. Cogn. Neurosci. 14, 1125-1138.

Hubl, D., Koenig, T., Strik, W. K. Garcia, L. M., and Dierks, T. (2007). Competition for neuronal resources: how hallucinations make themselves heard? Br. J. Psychiatry 190, 57-62.

Ishii, R., Shinosaki, K., Ikejiri, Y., Ukai, S., Yamashita, K., Iwase, M., MizunoMatsumoto, Y., Inouye, T., Yoshimine, T., Hirabuki, N., Robinson, S. E., and Takeda, M. (2000). Theta rhythm increases in left superior temporal cortex during auditory hallucinations in schizophrenia: a case report. Neuroreport 11, 3283-3287.

Jandl, M., Steyer, J., Weber, M., Linden D. E., Rothmeier, J., Maurer, K., and Kaschka, W. P. (2006). Treating auditory hallucinations by transcranial magnetic stimulation: a randomized controlled cross-over trial. Neuropsychobiology 53, 63-69.

Jeon, Y. W., and Polich, J. (2003). Metaanalysis of P300 and schizophrenia: patients, paradigms, and practical implications. Psychophysiology 40 684-701.

Kasai, K., Nakagome, K., Itoh, K., Koshida I., Hata, A., Iwanami, A., Fukuda, M. and Kato, N. (2002). Impaired cortical network for preattentive detection of change in speech sounds in schizophrenia: a high-resolution event-related potential study. Am. J. Psychiatry 159, 546-553.

Kindler, J., Hubl, D., Strik, W. K., Dierks, T., and Koenig, T. (2010). Resting-state
EEG in schizophrenia: auditory verbal hallucinations are related to shortening of specific microstates. Clin. Neurophysiol. doi: 10.1016/j. clinph.2010.10.042. [Epub ahead of print].

Lee, S. H., Wynn, J. K., Green, M. F., Kim, H., Lee, K. J., Nam, M., Park, J. K., and Chung,Y.C. (2006). Quantitative EEG and low resolution electromagnetic tomography (LORETA) imaging of patients with persistent auditory hallucinations. Schizophr. Res. 83, 111-119.

Line, P., Silberstein, R. B., Wright, J. J., and Copolov, D. L. (1998). Steady state visually evoked potential correlates of auditory hallucinations in schizophrenia. Neuroimage 8, 370-376.

Liu, Z., Tam, W. C., Xue, Z., Yao, S., and Wu, D. (2004). Positive and negative symptom profile schizophrenia and abnormalities in the P300 component of the event-related potential: a longitudinal controlled study. Psychiatry Res. 132, 131-139.

Marjerrison, G., Krause, A. E., and Keogh, R. P. (1968). Variability of the EEG in schizophrenia: quantitative analysis with a modulus voltage integrator. Electroencephalogr. Clin. Neurophysiol. 24, 35-41.

McCasland, J. S., and Konishi, M. (1981). Interaction between auditory and motor activities in an avian song control nucleus. Proc. Natl. Acad. Sci. U.S.A. 78, 7815-7819.

Paus, T., Marrett, S., Worsley, K., and Evans, A. (1996). Imaging motorto-sensory discharges in the human brain: an experimental tool for the assessment of functional connectivity. Neuroimage 4, 78-86.

Poulet, J. F., and Hedwig, B. (2002). A corollary discharge maintains auditory sensitivity during sound production. Nature 418, 872-876.

Reulbach, U., Bleich, S., Maihofner, C., Kornhuber, J., and Sperling, W. (2007). Specific and unspecific auditory hallucinations in patients with schizophrenia: a magnetoencephalographic study. Neuropsychobiology 55, 89-95.

Ropohl, A., Sperling, W., Elstner, S., Tomandl, B., Reulbach, U. Kaltenhauser, M., Kornhuber, J., and Maihofner, C. (2004). Cortical activity associated with auditory hallucinations. Neuroreport 15, 523-526.

Schall, U., Catts, S. V., Karayanidis, F., and Ward, P. B. (1999). Auditory eventrelated potential indices of frontotemporal information processing in schizophrenia syndromes: valid outcome prediction of clozapine therapy in a three-year follow-up. Int. J. Neuropsychopharmacol.2,83-93.

Sem-Jacobsen, C. W., Petersen, M. C., Lazarte, J. A., Dodge, H. W. Jr., and 
Holman, C.B. (1955).Intracerebral electrographic recordings from psychotic patients during hallucinations and agitation. Am. J. Psychiatry 112, 278-288.

Serafetinides, E. A., Coger, R. W., and Martin, J. (1986). Different methods of observation affect EEG measures associated with auditory hallucinations. Psychiatry Res. 17, 73-74.

Spencer, K. M., Niznikiewicz, M. A., Nestor, P. G., Shenton, M. E., and McCarley, R. W. (2009). Left auditory cortex gamma synchronization and auditory hallucination symptoms in schizophrenia. BMC Neurosci. 10, 85 .

Spencer, K. M., Salisbury, D. F., Shenton, M. E., and McCarley, R. W. (2008). Gamma-band auditory steady-state responses are impaired in first episode psychosis. Biol. Psychiatry 64, 369-375.

Sperry, R. W. (1950). Neural basis of the spontaneous optokinetic response produced by visual inversion. J. Comp. Physiol. Psychol. 43, 482-489.

Sritharan, A., Line, P., Sergejew, A., Silberstein, R., Egan, G., and Copolov, D. (2005). EEG coherence measures during auditory hallucinations in schizophrenia. Psychiatry Res. 136, 189-200.
Stevens, J. R., Bigelow, L., Denney, D., Lipkin, J., Livermore, A. H., Jr., Rauscher, F., and Wyatt, R. J. (1979). Telemetered EEG-EOG during psychotic behaviors of schizophrenia. Arch. Gen. Psychiatry 36, 251-262.

Stevens, J. R., and Livermore, A. (1982). Telemetered EEG in schizophrenia: spectral analysis during abnormal behaviour episodes. J. Neurol. Neurosurg. Psychiatry 45, 385-395.

Tiihonen, J., Hari, R., Naukkarinen, H., Rimon, R., Jousmaki, V., and Kajola, M. (1992). Modified activity of the human auditory cortex during auditory hallucinations. Am. J. Psychiatry 149, 255-257.

Turetsky, B., Colbath, E. A., and Gur, R. E. (1998). P300 subcomponent abnormalities in schizophrenia: II. Longitudinal stability and relationship to symptom change. Biol. Psychiatry 43, 31-39.

Uhlhaas, P. J., and Singer, W. (2010). Abnormal neural oscillations and synchrony in schizophrenia. Nat. Rev. Neurosci. 11, 100-113.

van Lutterveld, R., Oranje, B., Kemner, C., Abramovic, L., Willems, A. E., Boks,
M. P., Glenthoj, B. Y., Kahn, R. S. and Sommer, I. E. (2010). Increased psychophysiological parameters of attention in non-psychotic individuals with auditory verbal hallucinations. Schizophr. Res. 121, 153-159.

Von Holst, E., and Mittelstaedt, H. (1950). Das Reafferenzprinzip (Wechselwirkungen zwischen Zentralnervensystem und Peripherie). Naturwissenschaften 37, 464-476.

Whitford, T. J., Mathalon, D. H., Shenton, M. E., Roach, B. J., Bammer, R., Adcock, R. A., Bouix, S., Kubicki, M., De Siebenthal, J., Rausch, A. C. Schneiderman, J. S., and Ford, J. M. (2011). Electrophysiological and diffusion tensor imaging evidence of delayed corollary discharges in patients with schizophrenia. Psychol. Med. 41, 959-969.

Whitton, J. L., Moldofsky, H., and Lue, F (1978). EEG frequency patterns associated with hallucinations in schizophrenia and "creativity" in normals. Biol. Psychiatry 13, 123-133.

Youn, T., Park, H. J., Kim, J. J., Kim, M. S., and Kwon, J. S. (2003). Altered hemispheric asymmetry and positive symptoms in schizophrenia: equivalent current dipole of auditory mismatch negativity. Schizophr. Res. 59, 253-260.

Conflict of Interest Statement: The authors declare that the research was conducted in the absence of any commercial or financial relationships that could be construed as a potential conflict of interest.

Received: 11 February 2011; paperpending published: 25 April 2011; accepted:04 May 2011; published online: 16 May 2011.

Citation: van Lutterveld R, Sommer IEC and Ford JM (2011) The neurophysiology of auditory hallucinations-a historical and contemporary review. Front. Psychiatry 2:28. doi: 10.3389/fpsyt.2011.00028

This article was submitted to Frontiers in Schizophrenia, a specialty of Frontiers in Psychiatry.

Copyright (C) 2011 van Lutterveld, Sommer and Ford. This is an open-access article subject to a non-exclusive license between the authors and Frontiers Media SA, which permits use, distribution and reproduction in other forums, provided the original authors and source are credited and other Frontiers conditions are complied with. 